\title{
Interruption of classic CD40L-CD40 signalling but not of the novel CD40L-Mac-1 interaction limits arterial neointima formation in mice
}

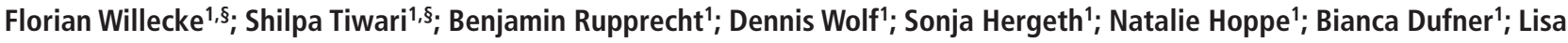 \\ Schulte'; Nataly Anto Michel'; Nora Bukosza'; Timoteo Marchini'; Markus Jäckel'; Peter Stachon'; Ingo Hilgendorf'; Katharina \\ Zeschky'; Rebecca Schleicher ${ }^{2}$; Harald F. Langer²; Constantin von zur Muhlen'; Christoph Bode'; Karlheinz Peter ${ }^{3}$; Andreas Zirlik ${ }^{1}$ \\ ${ }^{1}$ Atherogenesis Research Group, Heart Center Freiburg University, Freiburg, Germany; ${ }^{2}$ Department of Cardiovascular Medicine, University of Tübingen, Tübingen, Germany; ${ }^{3}$ Baker \\ IDI Heart and Diabetes Institute, Melbourne, Australia
}

\begin{abstract}
Summary
The co-stimulatory immune molecule CD40L figures prominently in a variety of inflammatory conditions including arterial disease. Recently, we made the surprising finding that CD40L mediates atherogenesis independently of its classic receptor CD40 via a novel interaction with the leukocyte integrin Mac-1. Here, we hypothesised that selective blockade of the CD40L-Mac-1 interaction may also retard restenosis. We induced neointima formation in C57/BL6 mice by ligation of the left carotid artery. Mice were randomised to daily intraperitoneal injections of either $\mathrm{CM7}$, a small peptide selectively inhibiting the CD40L-Mac-1 interaction, scM7, a scrambled control peptide, or saline for 28 days. Interestingly, cM7-treated mice developed neointima of similar size compared with mice receiving the control peptide or saline as assessed by computer-assisted analysis of histological cross sections. These data demonstrate that the CD40L-Mac- 1 interaction is not required for the development of restenosis. In contrast, CD40-deficient
\end{abstract}

\section{Correspondence to:}

Prof. Dr. Andreas Zirlik

Atherogenesis Research Group, Heart Center Freiburg University

Department of Cardiology and Angiology I

Hugstetter Str. 55, 79106 Freiburg, Germany

Tel.: +49 761270 34415, Fax: +4976127034426

E-mail: andreas.zirlik@universitaets-herzzentrum.de

These authors contributed equally to this work. mice subjected to carotid ligation in parallel, developed significantly reduced neointimal lesions compared with respective wild-type controls $\left(2872 \pm 843 \mu \mathrm{m}^{2}\right.$ vs $\left.35469 \pm 11870 \mu \mathrm{m}^{2}\right)$. Flow cytometry in CD40-deficient mice revealed reduced formation of platelet-granulocyte and platelet-inflammatory monocyte- aggregates. In vitro, supernatants of CD40-deficient platelet-leukocyte aggregates attenuated proliferation and increased apoptosis of smooth muscle cells. Unlike in the setting of atherosclerosis, CD40L mediates neointima formation via its classic receptor CD40 rather than via its recently described novel interaction with Mac-1. Therefore, selective targeting of CD40L-Mac-1 binding does not appear to be a favorable strategy to fight restenosis.

\section{Keywords}

Inflammation, CD40L, CD40, Mac-1, restenosis, neointima formation, mice

Received: August 12, 2013

Accepted after major revision: February 26, 2014

Prepublished online: March 20, 2014

http://dx.doi.org/10.1160/TH13-08-0653

Thromb Haemost 2014; 112: an

\section{Introduction}

An extensive body of experimental and clinical evidence implicates inflammation with neointimal hyperplasia and restenosis after coronary interventions $(1,2)$. Following injury to the vessel wall a network of inflammatory cellular and molecular regulatory pathways are activated. Vascular inflammation involves complex heterotypic interactions between endothelial cells, platelets, and inflammatory cells including neutrophils, monocytes, lymphocytes, and mast cells. Under normal circumstances, the cellular and molecular processes governing these responses mediate repair and vascular healing. In pathological conditions, however, dysregulation of inflammatory responses results in persistent inflammation and adverse arterial remodeling contributing to the development of clinical complications such as restenosis.
The CD40/CD40L dyad represents a crucial mediator of various inflammatory diseases, such as rheumatoid arthritis, transplant rejection, multiple sclerosis, and atherosclerosis $(3,4)$. CD40 ligand (CD40L, CD154), a member of the tumour necrosis factoralpha (TNFa) superfamily, is preferentially expressed on activated $\mathrm{CD}^{+} \mathrm{T}$-cells and activated platelets, but is also found on other haematopoietic and non-haematopoietic cells such as monocytes/ macrophages, endothelial cells (EC), and smooth muscle cells $(\mathrm{SMC})(5,6)$, cell types centrally involved in vascular disease and neointima formation. Mechanistically, CD40L induces proliferation and migration of smooth muscle cells (SMCs) by up-regulation of matrix metalloproteinase (MMP) 9 transcript and protein levels (7) and induces expression of key inflammatory cytokines such as monocyte chemoattractant protein (MCP)-1, interkeukin (IL)-1 $\beta$, IL-6, and IL-8 $(8,9)$. In vivo, CD40L impairs the function of peripheral blood angiogenic outgrowth cells and increases 
neointima formation after arterial injury (10) while anti-CD40L antibody treatment reduces neointima formation in mice subjected to vascular injury (11). CD40L expression on platelets is associated with late restenosis after percutaneous coronary intervention (12). However, assessment of neointima formation in CD40L-deficient mice gave ambivalent results (13).

The binding partner of CD40L, CD40 has also been implicated with neointima formation: CD40-deficient mice develop less neointima after ligation and wire injury (13-15) compared with respective wild-type controls. Both, in vitro and in vivo data suggest that CD40-induced neointima formation is mediated through its adaptor protein TNF receptor-associated factor 6 (TRAF6) in vascular SMCs (14) while the role of TRAF6 in classic atherogenesis is controversial and most likely cell type-dependent $(16,17)$. Besides binding to CD40, CD40L also interacts with integrins such as $\alpha 5 \beta 1$ (18) and $\alpha \mathrm{M} \beta 2$ (Mac-1) $(19,20)$. Mac-1 itself binds to multiple ligands such as glycoprotein (GP)Iba, ICAM-1, ICAM-2, and fibrinogen and is a crucial regulator of leukocyte recruitment by promoting firm adhesion of leukocytes to adherent platelets and fibrinogen (21). We identified the interaction of CD40L and Mac-1 as an alternative pathway for CD40L-mediated inflammation in atherogenesis (19). More recently, we identified the binding site of CD40L on Mac-1, the EQLKKSKT motif within the I-domain, and generated the selective peptide inhibitor $\mathrm{CM} 7$ that reduced peritoneal inflammation and inflammatory cell recruitment to the vascular bed in vivo (20). In addition, inhibition of CD40L-Mac-1 interaction abated atherosclerotic lesion development in low-density lipoprotein receptor (LDLr)-deficient mice. Mac-1 has also been associated with neointimal hyperplasia after vascular injury (22) and clinical studies showed that activation of circulating neutrophils (as determined by enhanced Mac-1 expression) coincides with late lumen loss and restenosis (23-26). Accordingly, Mac1-deficient mice developed decreased neointima formation following vascular injury most likely due to decreased leukocyte infiltration (27).

In the light of these findings suggesting a role for both CD40L and Mac-1 in neointima formation we hypothesised that inhibition of the novel CD40L-Mac1 interaction protects from neointima formation in mice.

\section{Methods}

\section{Mouse model of carotid artery ligation}

To evaluate the effect of CD40L-Mac-1 interaction on neointima formation, male C57BL/6 mice aged eight to 10 weeks were anesthetised on day 0 with $10 \%$ Ketamine and 2\% Xylazine. The carotid artery was ligated with silk suture (6-0) close to the left bifurcation to trigger neointima formation as described previously (28). Mice then received daily intraperitoneal (i.p.) injections of the peptide inhibitor cM7 $(4 \mu \mathrm{g} / \mathrm{kg})$, its scrambled control scM7 (4 $\mu \mathrm{g} / \mathrm{kg}$, Peptide Specialty Laboratory, Heidelberg, Germany) or saline. After 28 days on normal diet all mice were euthanised and the carotid arteries were harvested. To evaluate the role of CD40 deficiency on neointima formation, CD40-deficient mice (Jackson
Laboratories, Bar Harbor, ME, USA) were subjected to the same procedure and compared with male wild-type C57BL/6 mice (Jackson Laboratories). All mice were housed under specific pathogen-free conditions. All procedures were approved by the Animal Care Committee of the University of Freiburg and the local authorities (Regierungspräsidium Freiburg, Az. 35-9185.81/G-12/76).

\section{Intravital microscopy}

Male C57BL/6 mice aged eight to 10 weeks were anesthetised on day 0 with $10 \%$ Ketamine and 2\% Xylazine. The carotid artery was ligated with silk suture (6-0) close to the left bifurcation as described above. Following surgery, all mice were treated i.p. with the peptides cM7 or scM7 (4 $\mu \mathrm{g} / \mathrm{kg}$, dissolved in sterile saline) for six days. On the sixth post-surgery day mice received i.p. injections of $200 \mathrm{ng}$ of murine TNF $\alpha$ (R\&D Systems, Minneapolis, MN, USA) 4 hours (h) before intravital microscopy. Intravital microscopy was performed as described previously (29). Mice were placed on a heating pad to maintain body temperature. Videos were taken with an intravital microscope (AxioScope Vario, Carl Zeiss, Jena, Germany) fitted with a saline immersion objective (WPlanAPOCHROMAT 20x/1,0DIC IR, Carl Zeiss) and a high sensitivity camera system (AxioCam MRm, Carl Zeiss) for 30 seconds (s) each. Rolling leukocyte flux was defined as the number of leukocytes moving at a velocity less than erythrocytes. Adherent leukocytes were defined as cells that remained stationary for at least 30 s. Rolling leukocyte flux, adhering flux were quantified by blinded investigators.

\section{Preparation and histology of ligated carotid arteries}

At 28 days after carotid artery ligation, mice were euthanised and the arterial system was perfused through the left cardiac ventricle with saline and subsequently with $1 \%$ agarose. Carotid arteries were removed, fixed in $10 \%$ paraformaldehyde, and then embedded in paraffin. For histologic analysis cross-sections $(5 \mu \mathrm{m})$ were cut at $250 \mu \mathrm{m}$ intervals throughout the common carotid artery starting at the bifurcation. For each of the eight levels, a crosssection was stained with Movat. Movat-stained cross-sections were used for morphometric analysis of the lumen area, intimal area (the area within the internal elastic lamina [IEL] minus the lumen area), medial area (defined as the area within the external elastic lamina [EEL] minus the area within the IEL), and total vessel area (area encompassed by the EEL). Intimal volumes were determined by multiplying intimal areas with the distance analyzed. Luminal and total vessel volumes were determined by multiplying lumen and total vessel areas including eight levels.

\section{Immunohistochemistry}

In addition to the Movat staining, sections were stained with antimac3 (1:1000, BD Pharmingen, San Jose, CA, USA) followed by a biotinylated rabbit anti-rat secondary antibody (BA-4001, Vector Laboratories, Burlingame, CA, USA) for macrophages. For detec- 
tion of smooth muscle cells, sections were stained with anti$\alpha$-actin-FITC-conjugated antibody (1:1000; Sigma Diagnostics, St. Louis, MO, USA) followed by anti-FITC-biotin conjugated antibody (Sigma) as described previously (30). Slides were developed with AEC Substrate Chromogen (Dako, Glostrup, Denmark).

Furthermore, ICAM-1 positive cells were analysed by staining sections with anti-ICAM-1 antibody (1:100, Abcam, Cambridge, UK), followed by the biotinylated goat anti-rabbit secondary antibody (1:200, Vector). VCAM-1 positive cells were stained by using anti-VCAM-1 antibody (1:100, Santa Cruz Biotechnologies, Santa Cruz, CA, USA) and biotinylated rabbit anti-goat (1:200, Vector) as the secondary antibody. Mac-3, $\alpha$-actin, VCAM-1 and ICAM-1 positive cells per intimal area were determined by computer-assisted image quantification (ImagePro, Media Cybernetics, Rockville, MD, USA).

\section{Flow cytometry}

Flow cytometry was performed as previously described using FACSCantoII (Becton Dickinson) (20). Blood samples (100 $\mu \mathrm{l} /$ mouse) were collected from the retroorbital plexus after anesthesia with isoflurane. Cells were pre-incubated with mouse FcBlock (CD16/32, eBioscience, San Diego, CA, USA). Antibodies included CD11b-FITC, CD115-PE, Ly6C/G (Gr1)-APC, CD4-Alexa488, CD8-PE, CD3-APC, and CD41-FITC (all eBioscience). The leukocyte population was set in a forward angle (FSC) vs right angle scatter (SSC). Blood monocytes were quantified by gating for $\mathrm{CD} 11 \mathrm{~b}$ and $\mathrm{CD} 115$ positive cell population. Inflammatory monocytes were selected by additional gating for Gr1 positive cells. For analysis of T-cells, histogram dot plots were used to select for lymphocytes and gate for CD3-positive cells. To identify the CD8- and CD4- positive cells, CD3 expressing cells were set in a CD8-PE vs CD4-Alexa488 staining. Platelet-leukocyte aggregates were detected by additional gating for CD41 in its respective cell population (Suppl. Figures 5 and 6 for representative FACS plots, available online at www.thrombosis-online.com). Cytometric data was analyzed employing FlowJo software (Tree Star Inc., Ashland, OR, USA).

For assessment of platelet activation C57BL/6 mice were treated i.p. with cM7 or scM7 (both $4 \mu \mathrm{g} / \mathrm{kg}$ body weight) for four days. Then Murine platelet preparations were obtained using a modified protocol (31). Briefly, blood was drawn from the retroorbital plexus via heparinised capillaries into a tube with $300 \mu \mathrm{l}$ ACD buffer (12.5 g sodium-citrate, $2 \mathrm{~g}$ citric acid, 10 g glucose in $500 \mathrm{ml}$ Aqua dest.; $\mathrm{pH}$ 4.7). To prevent platelet activation, apyrase and prostaglandin were used. To activate platelets, isolated platelets $\left(1 \times 10^{6}\right)$ were stimulated at room temperature with $10 \mu \mathrm{M}$ ADP or 0.2 $\mathrm{U} / \mathrm{ml}$ Thrombin. The activation of platelets was monitored by flow cytometry using anti-mouse P-selectin antibody (Wug.E9, emfret Analytics, Eibelstadt, Germany) and anti-mouse GPIIb/IIIa antibody (JON/A, emfret Analytics). The staining was performed according to the manufacturer's recommendations and samples were analysed with a FACS-Calibur flow cytometer (Becton-Dickinson, Heidelberg, Germany).

\section{Assessment of plasma cytokines and matrix metallo- proteinases}

Blood was collected by intracardial puncture from mice 28 days after ligation of the carotid artery. Plasma concentrations of IL-6, IL-10, IL-12p70, MCP-1 and TNFa were determined by the Cytometric Bead Array (CBA, BD Biosciences) as described previously (32). Plasma levels of CXCL4 were determined by ELISA (Mouse CXCL4/PF4, DuoSet) according to the manufacturer's instructions. Plasma concentrations of MMP2 and MMP9 were measured by ELISA using the MMP2 Mouse ELISA Kit (Abcam) and Mouse Total MMP9 DuoSet (R\&D).

\section{Cell culture}

For ex vivo analysis of platelet-leukocyte interaction retroorbital blood was collected in enoxaparin/phosphate-buffered saline (PBS) $(100 \mu \mathrm{g} / \mathrm{ml}) 1: 1$ and centrifugated at $100 \mathrm{~g}$ x 5 minutes (min) at $37^{\circ} \mathrm{C}$. Platelets were taken off and counted in a Neubauer chamber. The red pellet was lysated with RBC Lysis Buffer on ice and washed with cool PBS. Leukocyte were counted and incubated with platelets in ratio of $1: 5$ for $20 \mathrm{~min}$ at $37^{\circ} \mathrm{C}$ followed by centrifugation at $1,500 \mathrm{~g}$ for $3 \mathrm{~min}$. Platelet-leukocyte aggregates were quantified by flow cytometry as described above.

To study the effect of platelet-leukocyte aggregates on SMC proliferation, murine SMCs (ATCC, CRL-2797, cell line from C57BL/6 mice) were grown in 96-well plates to $80 \%$ confluency. The murine smooth muscle cells were then incubated with the supernatants of platelet-leukocyte aggregates for $12 \mathrm{~h}$. Apoptosis and proliferation of smooth muscle cells was measured by Apo-ONE ${ }^{\circledast}$ Homogeneous Caspase-3/7 Assay (Promega, Madison, WI, USA) and BrdU ELISA (Roche, Basel, Switzerland) according to the instructions of the manufacturers.

\section{Statistical analysis}

Data are presented as mean \pm SEM. Significance was measured with the unpaired Student's t-test or analysis of variance (ANOVA), followed by Newman-Keuls Multiple Comparison Test where appropriate. P-values $<0.05$ were considered significant.

\section{Results}

\section{Inhibition of the CD40L-Mac-1 interaction attenuates endothelial leukocyte adhesion and rolling after carotid ligation without affecting platelet activation}

We previously showed in vitro and in vivo that leukocyte adhesion in mice is impaired after inhibition of the CD40L-Mac1 interaction with the peptide inhibitor cM7 $(20,33)$. We now studied the effect of cM7 on leukocyte adhesion and rolling after carotid ligation in mesenterial venules. We detected a significant decrease in leucocyte adhesion and rolling in cM7 treated mice, corroborating that inhibition of CD40L-Mac-1 impairs leukocyte adhesion (Suppl. Figure 1A, available online at www.thrombosis-online. 


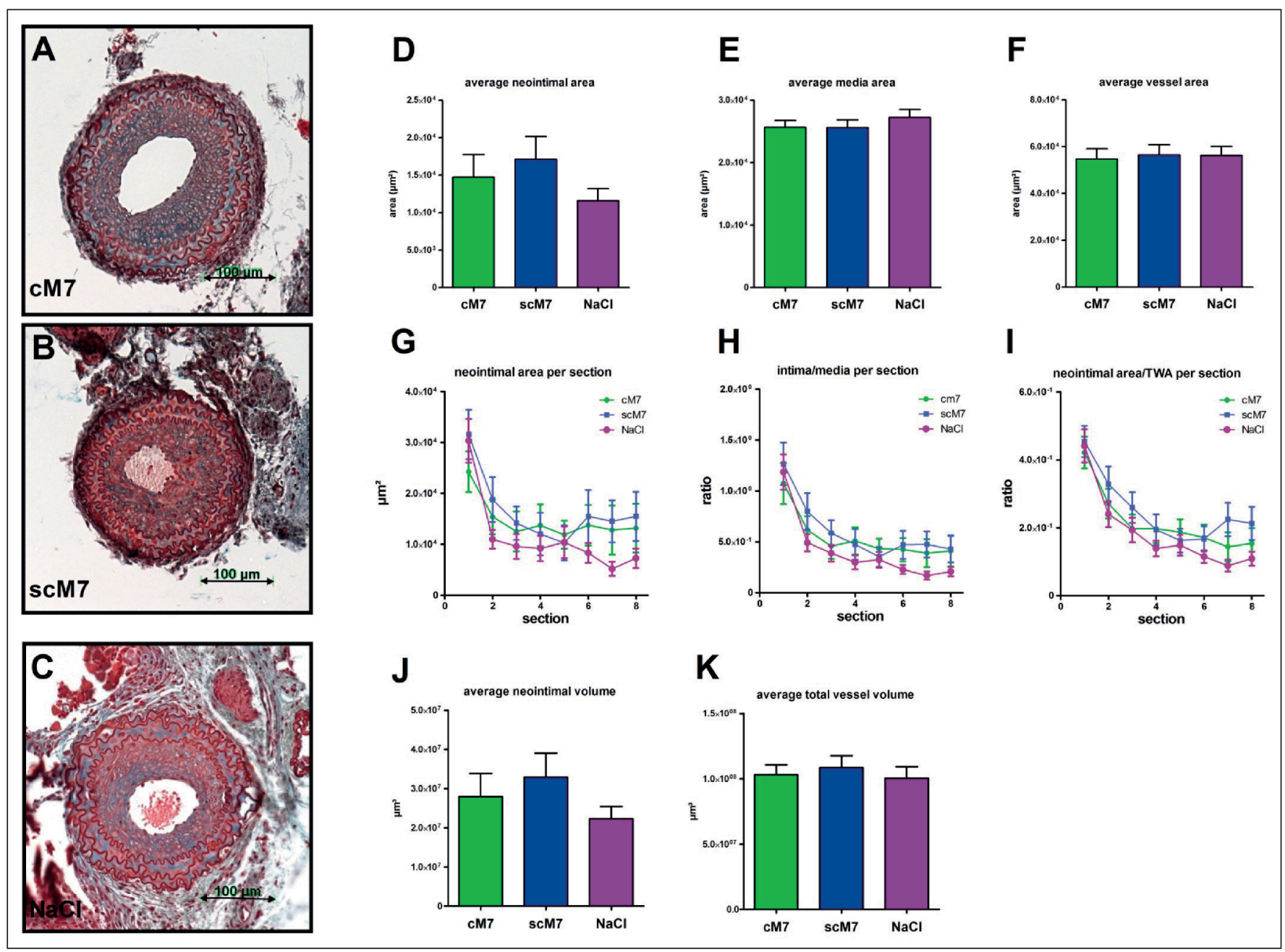

Figure 1: Neointimal formation in cM7-, scM7- and NaCl-treated mice 28 days after carotid ligation. MOVAT-stained representative crosssections of cM7-, scM7- and NaCl-injected mice are displayed (A-C). $n=20$, 18 , and 17 , respectively. The average area of the neointima (D), media (E), and total vessel (F) of eight cross sections ( $250 \mu \mathrm{m}$ intervals) were analyzed

com). Since blockade of CD40L can increase thrombus formation $(34,35)$ we also measured platelet activation by quantifying the expression of activated GPIIbIIIa (clone JON-A) or P-selectin on platelets of animals treated with $\mathrm{cM} 7$ or scM7 control using flow cytometry (Suppl. Figure 2, available online at www.thrombosisonline.com). These data suggest that our peptide targeting the CD40L-Mac-1 interaction has no relevant effect on the tested platelet activation markers. For instance, no significant difference in platelets positive for activated GPIIbIIIa or double positive for activated GPIIbIIIa and P-selectin was detected after exposure to cM7 in comparison to scrambled control peptide. In a previous study we also showed that inhibition of the CD40L-Mac-1 interaction does not affect thrombus formation (20). and displayed over all eight consecutive cross sections as neointimal area/ section, neointima/media ratio per section and neointima/total wall area (G-I). In addition, the average neointimal volume and the average vessel volume was calculated (J, K). Error bars represent SEM.

\section{Inhibition of the CD40L-Mac-1 interaction does not attenuate neointimal hyperplasia}

To determine the possible role of CD40L-Mac-1 interaction in neointima formation, C57BL/6J mice underwent ligation of the left carotid artery followed by treatment with the specific inhibitor cM7 of the CD40L-Mac-1 interaction, the scrambled control scM7, or saline for 28 days. Body weight did not differ between the groups at beginning and at the end of the study ( $>$ Table 1 ). Analysis of consecutive Movat-stained cross-sections revealed that cM7-treated mice developed equal amounts of neointimal hyperplasia compared with both, scM7- and saline-treated mice, suggesting no role of the CD40L-Mac-1 interaction in restenosis ( $>$ Figure 1). Since both CD40L and Mac-1 play a role in the attraction of macrophages and proliferation of smooth muscle cells we analysed macrophage and smooth muscle cell content in carotid cross sections. In line with our data on lesion size, immunohis- 


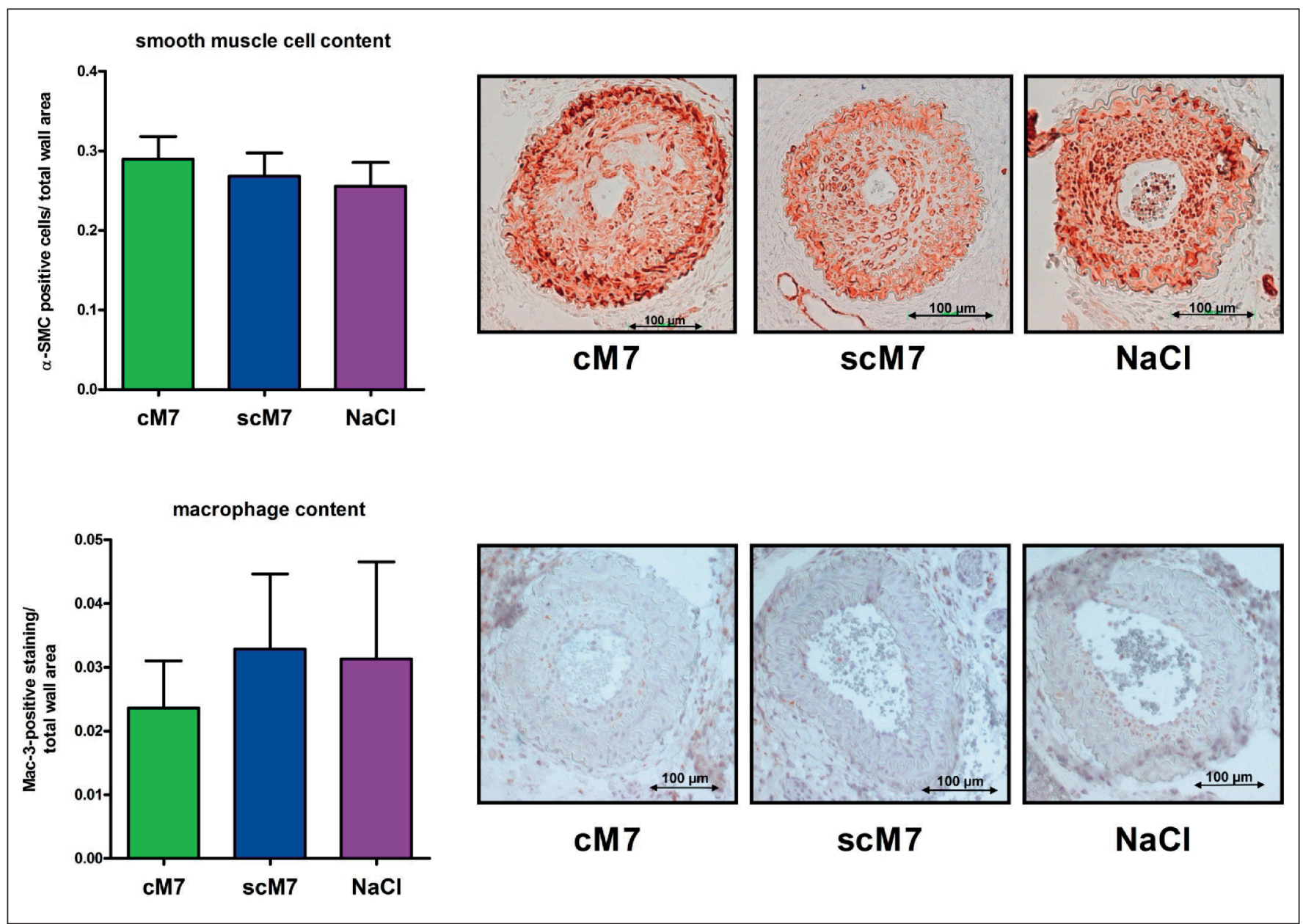

Figure 2: Smooth muscle cell and macrophage cell content of the vessel wall in cM7-, scM7-, and NaCl- treated mice 28 days after carotid ligation. Smooth muscle cell and macrophage content was quantified by the amount of ?-SMC-positive cell and Mac-3-positive cells, respectively, in the total wall area. $\mathrm{N}=15$ per group. Error bars represent SEM.

tochemistry showed similar content of both, macrophages and SMCs, in the ligated carotid arteries of treated and untreated animals ( $\triangleright$ Figure 2). Also, we did not detect a difference in endothelial cell activation as assessed by staining for ICAM-1 and VCAM-1 (Suppl. Figure 1B, available online at www.thrombosisonline.com).

\section{cM7 does not alter T-lymphocyte, monocyte or leukocyte-platelet aggregate numbers}

Since both, CD40L and Mac-1 are potentially functionally relevant for the interaction of leukocyte subsets and platelets; we analysed blood cells by flow cytometry after carotid ligation and 28 days of treatment with $\mathrm{cM} 7, \mathrm{scM} 7$, or saline ( $>$ Figure 3). We did not detect a significant difference in the number of T-lymphocytes, inflammatory monocytes, or monocyte/granulocyte-platelet aggregates ( Figure 3A-G). Plasma cytokine levels (interferone [IFN] $\gamma$, IL-10, IL-12, IL-6, MCP-1 and TNFa), MMP2 and MMP9 and CXCL4 (PF4) were not affected by CD40L-Mac-1 inhibition
(Suppl. Figure 1C-E, available online at www.thrombosis-online. com).

Table 1: Baseline characteristics of mice.

\begin{tabular}{|c|c|c|c|}
\hline & cM7 & scM7 & $\mathrm{NaCl}$ \\
\hline Number of mice & 24 & 22 & 19 \\
\hline \multirow[t]{2}{*}{$\begin{array}{l}\text { Body weight (g) } \\
\text {-before ligation } \\
\text {-after } 28 \text { days }\end{array}$} & $\begin{array}{l}22.4 \pm 0.3 \\
25.3 \pm 0.3\end{array}$ & $\begin{array}{l}22.6 \pm 0.2 \\
25.3 \pm 0.3\end{array}$ & $\begin{array}{l}22.7 \pm 0.3 \\
25.4 \pm 0.4\end{array}$ \\
\hline & CD40 & C57BI/6J & \\
\hline Number of mice & 10 & 10 & \\
\hline $\begin{array}{l}\text { Body weight (g) } \\
\text {-before ligation } \\
\text {-after } 28 \text { days }\end{array}$ & $\begin{array}{l}24.0 \pm 0.5 \\
25.8 \pm 0.6\end{array}$ & $\begin{array}{l}23.9 \pm 0.2 \\
26.0 \pm 0.4\end{array}$ & \\
\hline
\end{tabular}




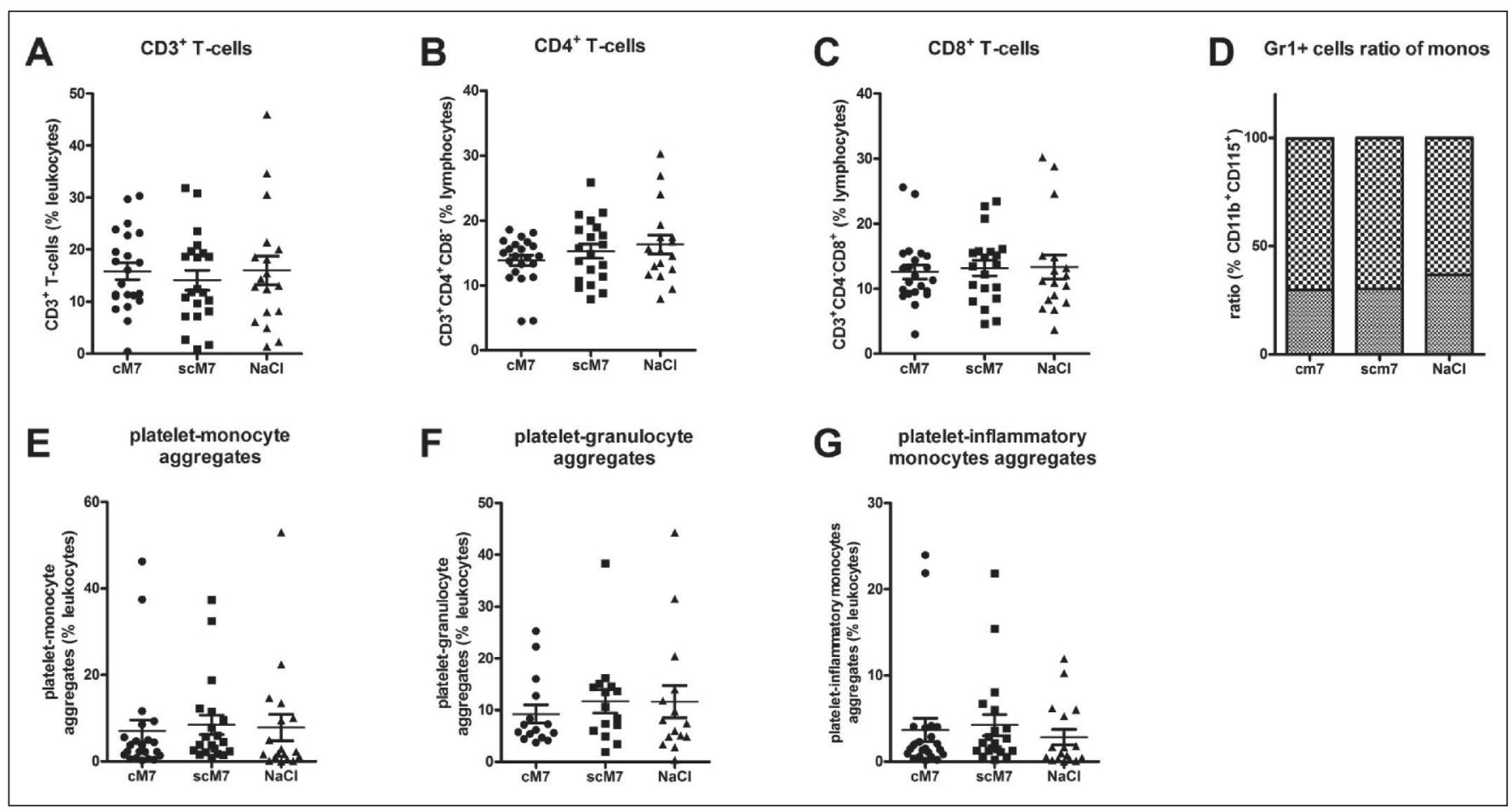

Figure 3: FACS analysis in cM7-, scM7-, and NaCl-treated mice. Relative amounts of circulating T-cells and subsets (A), CD4 ${ }^{+}$helper T-cells (B), CD8 ${ }^{+}$killer T-cells (C), inflammatory monocytes (D), platelet-monocyte aggregates (E), platelet-granulocyte aggregates (F) and platelet-inflammatory monocytes aggregates $(\mathrm{G})$, are displayed. $\mathrm{N}$ as indicated. Error bars represent SEM.

\section{CD40-deficiency diminishes neointima formation}

Based on our finding that the novel CD40L-Mac-1 interaction did not contribute to neointima formation in our model, we tested whether $\mathrm{CD} 40$ as the classic CD40L receptor is important in this scenario. Therefore, we studied the effect of CD40 deficiency on neointima formation. Neointima formation in CD40-deficient mice was significantly impaired compared with respective controls ( $>$ Figure 4). Of note, the average vessel area was also significantly reduced in CD40-deficient mice ( Figure 4E). This suggests an impaired remodelling capacity in the ligated arteries of $\mathrm{CD} 40$-deficient mice as the medial area and the average total lumen volume were similar in both groups ( $>$ Figure $4 \mathrm{~B}$ and J) (13).

\section{CD40 deficiency decreases platelet-inflammatory monocyte and -granulocyte aggregates and plasma levels of CXCL4 (PF4)}

Since CD40 is abundantly expressed by monocytes and platelets, both playing an important role in atherosclerosis and neointima formation (2), we further characterised CD40-deficient mice by flow cytometry ( $\$$ Figure 5 ). T-cell numbers were similar between CD40 knockout mice and wild-type mice, although there was a tendency towards more CD8-positive T-cells in CD40-deficient mice ( Figure $5 \mathrm{~A}-\mathrm{C})$. The number of inflammatory monocytes tended to decrease $(\mathrm{p}=0.06)$ in CD40-deficient mice $(\triangleright$ Figure
5D). The number of granulocytes and inflammatory monocytes attached to platelets decreased significantly in CD40-deficient mice ( Figure 5E-G). To test whether the decreased platelet-leukocyte aggregation is caused by a lack of CD40 on platelets or $\mathrm{CD} 40$ on leukocytes we co-cultured platelets and leukocytes isolated from $\mathrm{C} 57 \mathrm{BL} / 6$ and $\mathrm{CD} 40$-deficient mice in vitro and measured the formation of platelet-leukocyte aggregates. Formation of platelet-leukocyte aggregates were significantly reduced when either only leukocytes or both platelets and leukocytes were CD40-deficient, suggesting that CD40 on leukocytes mediates formation of these aggregates ( Figure 6A). Plateletmonocyte aggregates were also significantly decreased when both platelets and leukocytes were CD40-deficient (Suppl. Figure 4A, available online at www.thrombosis-online.com). In contrast to our in vivo data platelet-granulocyte aggregates were unchanged (Suppl. Figure 4B, available online at www.thrombosis-online. com). Whereas $\mathrm{CD} 40$ deficiency did not change inflammatory cytokines like IL-6, IL-12, TNFa, MCP- 1 and IFN $\gamma$ or plasma levels of MMP2 and MMP9 we detected a significant reduction in plasma levels of CXCL4 (PF4) in CD40-deficient mice after carotid ligation (Suppl. Figure 3A, available online at www.thrombosisonline.com). 


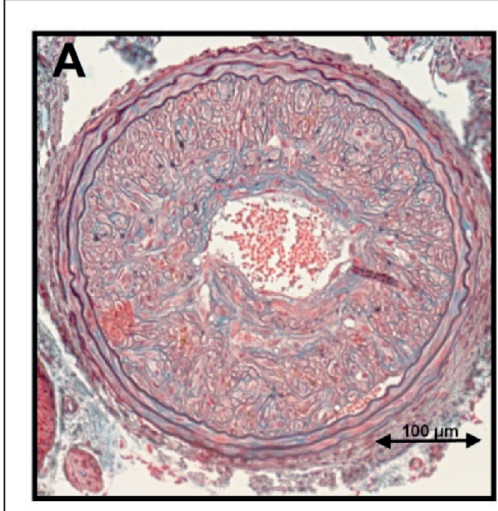

C57BL/6

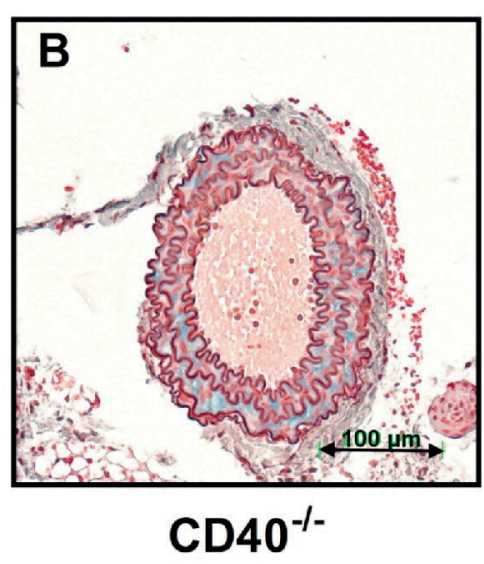

$F$
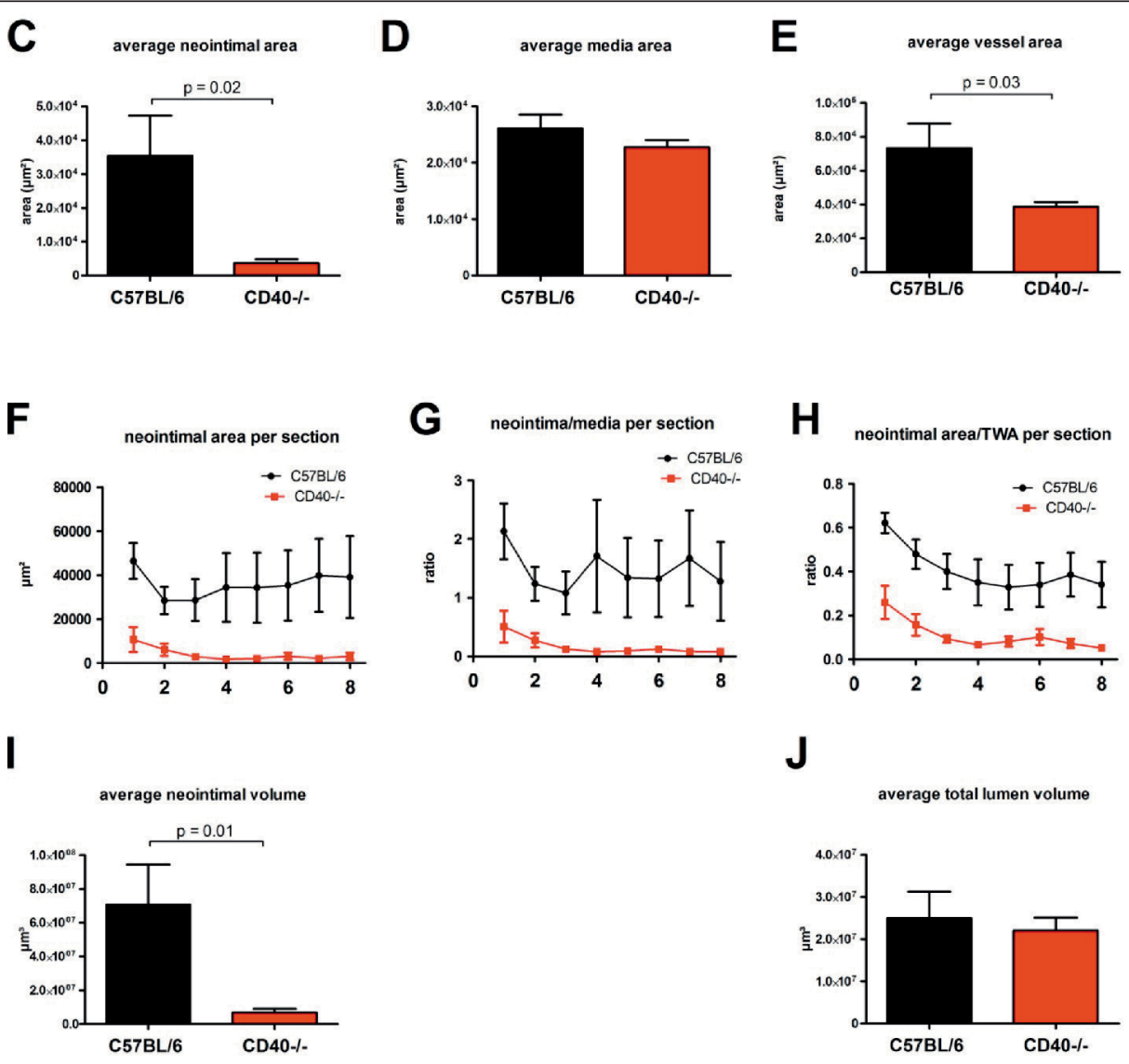

J

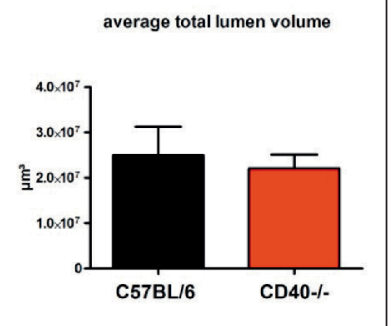

Figure 4: Neointimal formation in CD40-deficient mice verses respective control mice 28 days after carotid ligation. MOVAT-stained representative cross-sections of CD40-deficient and C57BI/6 control mice are displayed (A, B). N = 10 per group. The average area of the neointima (C), media (D), and total vessel (E) of eight cross sections ( $250 \mu \mathrm{m}$ intervals) were analyzed and displayed over all eight consecutive cross sections as neointi$\mathrm{mal}$ area/section, neointima/media ratio per section and neointima/total wall area $(\mathrm{F}-\mathrm{H})$. In addition, the average neointimal volume and the average vessel volume was calculated $(\mathrm{I}, \mathrm{J})$. N = 10 per group. Error bars represent SEM.

\section{CD40-deficient platelet-leukocyte aggregates attenuate smooth muscle cell proliferation and increase apoptosis}

SMC proliferation is a key event in neointima formation. To elucidate the role of platelets-leukocytes aggregates in smooth muscle cell proliferation we cultured a murine SMC cell line with supernatants of platelets-leukocytes aggregates from either C57BL/6 or CD40-deficient mice. Interestingly, SMC proliferation decreased and SMC apoptosis increased significantly when incubated with supernatants of CD40-deficient platelet-leukocyte aggregates, suggesting that CD40 can mediate SMC proliferation and apoptosis via platelet-leukocyte aggregates ( Figure 6B and C).

\section{Discussion}

This study elucidates the role of two important receptors of CD40L, CD40 and Mac-1, in neointima formation in response to carotid ligation. We recently discovered a new interaction between
$\mathrm{CD} 40 \mathrm{~L}$ and the $\beta 2$-integrin receptor Mac- 1 and showed that specific inhibition of the CD40L-Mac-1 interaction by the peptide inhibitor $\mathrm{cM} 7$ attenuated leukocyte recruitment, inflammation, and atherogenesis in mice (20). Of note, cM7 does not interfere with CD40L's immune and hemostatic functions (e.g. platelet activation and thrombus formation) - a side effect seen with other inhibitors of CD40L $(34,35)$. In accord, we detected decreased adhesion and rolling of leukocytes after carotid ligation in cM7-treated mice, whereas platelet activation was not significantly affected (Suppl. Figures $1 \mathrm{~A}$ and $2 \mathrm{~A}-\mathrm{C}$, available online at www.thrombosis-online. com).

Since a solid body of evidence suggests a role of CD40L and Mac-1 in neointima formation $(7,10,11,13,22,27)$ we hypothesised that specific inhibition of the CD40L-Mac-1 interaction with preservation of the CD40L/CD40 interaction - would reduce neointima formation in a mouse model of carotid ligation. In contrast to our initial hypothesis, neointima formation in mice treated with the specific CD40L/Mac-1 peptide inhibitor cM7 did not reduce neointima formation compared with mice treated with the scrambled control peptide scM7 or saline. In addition, we also 


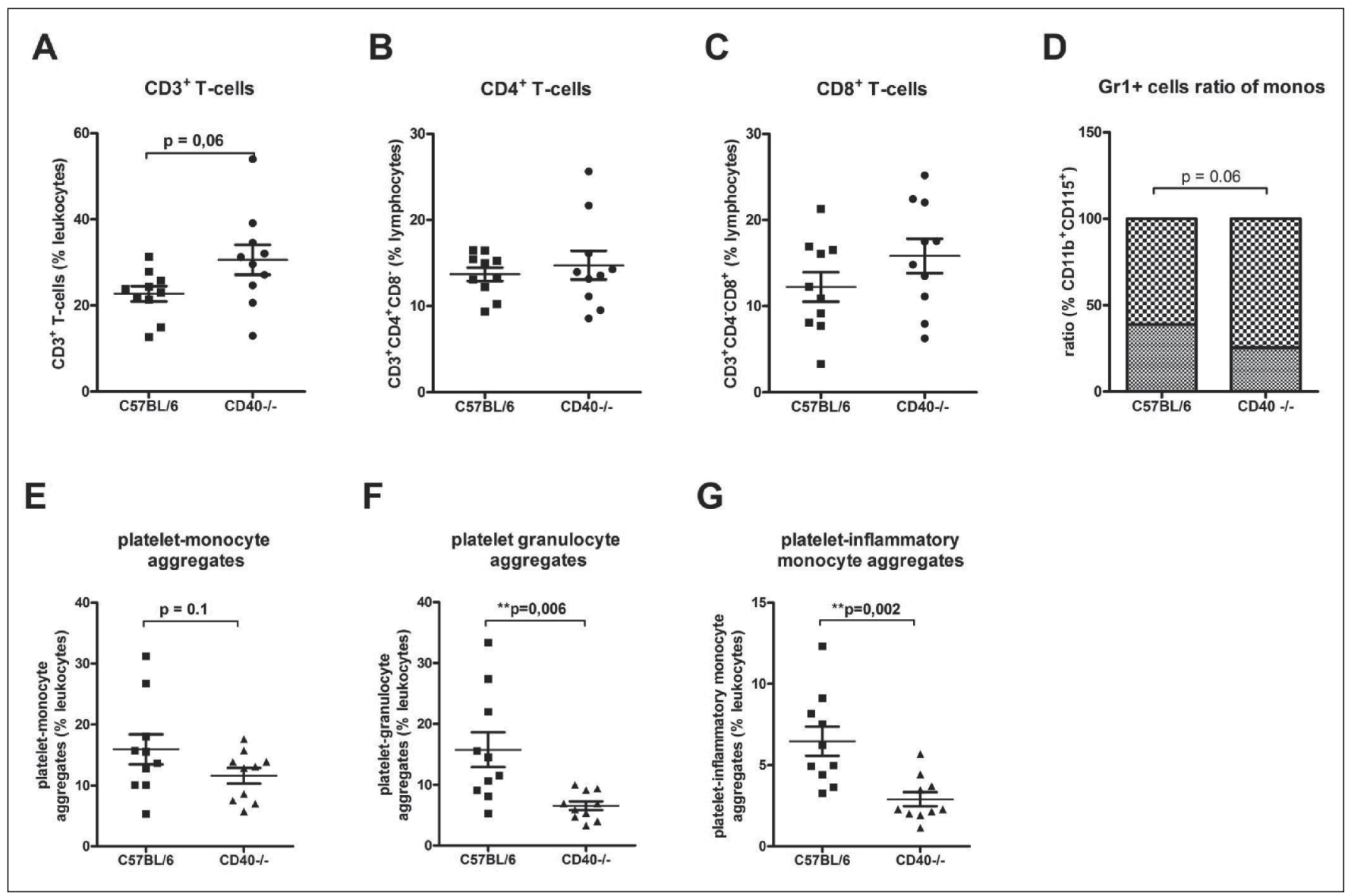

Figure 5: FACS analysis of peripheral blood in CD40-deficient and C57BL/6 mice: Relative amounts of circulating T-cells (A), CD4+ helper T-cells (B), $\mathrm{CD}^{+}$killer T-cells (C), inflammatory monocytes (D), platelet-monocyte aggregates (E), platelet-granulocyte aggregates (F) and platelet-inflammatory monocytes aggregates $(\mathrm{G})$, are displayed. $\mathrm{N}=10$ per group. Error bars represent SEM.

A

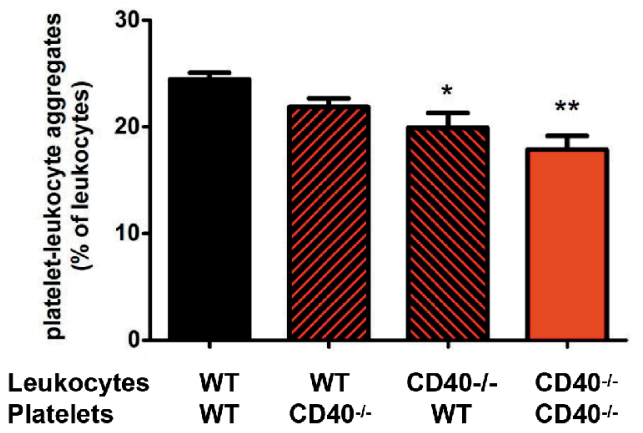

B



C

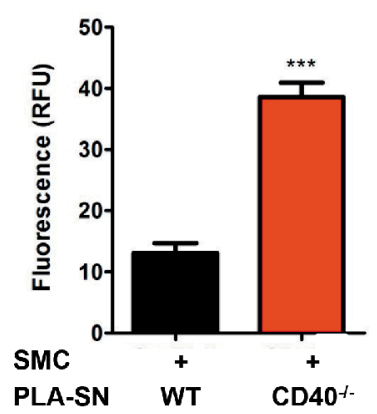

Figure 6: CD40 on leukocytes contributes to the formation of platelet-leukocyte aggregates. Platelets and leukocytes from CD40-deficient $\left(\mathrm{CD} 0^{-1-}\right)$ and $\mathrm{C} 57 \mathrm{BL} / 6$ mice (WT) were co-incubated as indicated and formation of platelet-leukocyte aggregates were assessed by flow cytometry (A). $N=6 /$ group. ${ }^{*} p<0.05,{ }^{* *} p<0.001$ vs. WT/WT. Supernatants of CD40-deficient platelet-leukocyte aggregates attenuate smooth muscle cell proliferation and increase apoptosis. A murine SMC line was stimulated with supernatants of platelet-leukocyte aggregates (PLA-SN) of either C57BL/6 mice (WT) or CD40-deficient (CD40-1-) mice. Proliferation of SMC was determined using a BrdU-based ELISA (B) and the rate of apoptosis was determined using Apo-ONE $\circledast$ Assay (C). $N=3$ /group. ${ }^{*} p<0.05,{ }^{* * *} p<0.001$ vs. WT. Error bars represent SEM. 
studied whether the inhibition of the CD40L/Mac-1 interaction had an effect on lesion composition, specifically on macrophages and SMC content in the neointima. Neointima formation is characterised by both, proliferation of SMCs and invasion of macrophages (2). Mac-1 is expressed on macrophages and in the vasculature. CD40L is also expressed on endothelial cells, macrophages, platelets, and smooth muscle cells (21). There are contradictory reports about the role of CD40L in SMC proliferation and migration in vitro. Chai et al. reported that CD40L increases SMC proliferation and migration (7), whereas Hermann et al. showed that CD40L does not stimulate SMC proliferation and migration (36). In our study both SMC and macrophage content was not altered in anti-Mac-3- and anti-aSMC-stained cross sections of lesions of mice treated with $\mathrm{cM} 7, \mathrm{scM} 7$, or saline, arguing against a role of CD40L/Mac-1 interaction for SMC and macrophage proliferation and migration after carotid ligation. Treatment with the CD40L/Mac-1 inhibitor cM7 did not alter the number of T-cells, monocytes, and platelets in blood compared with treatment with scrambled control or saline.

Since the inhibition of CD40L/Mac-1 interaction does not ablate neointima formation we hypothesised that neointima is rather mediated through an interaction of CD40L with its predominant receptor CD40. Indeed, neointima proliferation was markedly reduced in CD40-deficient mice compared with wild-type mice following carotid ligation. At the same time - as seen in the study by Donners et al. (13) - the average vessel area was also markedly reduced in CD40-deficient mice, revealing an overall impaired remodelling capacity in these mice after carotid ligation. There are several studies that previously evaluated the role of CD40 and CD40L in a variety of restenosis models: Blocking CD40L with an anti-CD40L monoclonal antibody attenuated neointima formation (11). Infusion of recombinant CD40L-accelerated neointimal progression in the carotid artery after wire injury (10). On the other hand, using flexible collars to induce neointimal thickening in carotid arteries in mice, Remskar et al. showed that CD40L ablation induced a more pronounced neointima formation. A possible explanation might be that CD40L deficiency reduces IFN $\gamma$, a cytokine that has anti-proliferative effects on SMC (37). Donners et al. reported that after wire injury, neointima formation in CD40L-deficient mice was similar compared with wild-type mice. In contrast, the same authors and another group reported that $\mathrm{CD} 40$-deficient mice displayed a marked reduction in neointimal proliferation and a reduction in total vessel volume $(13,14)$. Both studies support a key role for downstream CD40 signaling through TRAF6 in neointimal formation. As a possible explanation for the reduced neointima formation Donners et al. report a tendency towards decreased matrix-degrading protease activity (MMP9 and MMP2). In our study plasma levels of MMP9 and MMP2 were similar in CD40-deficient mice vs C57BL/6 mice after carotid ligation (Suppl. Figure 3A, available online at www. thrombosis-online.com). However, this does not rule out locally increased matrix-degrading protease activity at the side of the carotid ligation.

The fact that genetic deficiency in CD40 results in a phenotype in this model is not in contradiction to the absence of an effect for
What is known about this topic?

- CD40L and its ligands CD40 and Mac-1 have been implicated to play a role in neointima and restenosis formation.

- We have recently developed an inhibitor of the CD40L-Mac-1 interaction to study its role in inflammatory diseases like atherosclerosis and restenosis.

- Selective inhibition of the CD40L-Mac-1 interaction limits atherosclerosis in mice.

What does this paper add?

- Deficiency of the CD40L binding partner CD40 reduces neointima formation, suggesting that the classic CD40L/CD40 interaction mediates neointima formation rather than the CD40L-Mac-1 interaction. Future studies should therefore focus on downstream targets of the CD40-CD40L interaction to prevent restenosis.

- CD40 mediates platelet-leukocyte aggregation and smooth muscle cell proliferation in vitro.

the CD40L-Mac-1 inhibition either: a) these are two very different pathways affecting distinct functions, e.g. inflammatory cell recruitment in the case of CD40L-Mac-1 vs co-stimulation dependent- and independent pro-inflammatory effects in the case of CD40, b) even CD40 may have pro- and anti-inflammatory effects depending on its contribution to classic co-stimulation, direct gene expression in non-leukocytic cells or anti-inflammatory feedback mechanisms (e.g. CD40 expression on T cells) in different in vivo models. In that respect it is also noteworthy that while we previously showed that overall genetic deficiency of CD40 in LDLR KOs does not affect atherosclerosis (19), Lutgens et al. showed that the specific deletion of the CD40-TRAF6 interaction limits atherosclerosis in the same model contributing to the complexity of pathways (17).

Since both, leukocytes and platelets are major components of neointima formation we further characterised the cellular components of the blood in these mice after carotid ligation. We observed a significant decrease of monocyte- and granulocyte-platelet aggregates in CD40-deficient mice providing a possible explanation for a decreased neointima formation in these mice. In addition, we detected a decrease of CXCL4 (PF4) in the plasma of CD40-deficient mice after ligation of the carotid artery (Suppl. Figure 3B, available online at www.thrombosis-online.com). PF4 is released in large amounts at sites of vascular injury and has been implicated to play an important role in thrombosis (38). Platelet activation and thrombus formation are the first steps following flow cessation as it takes place after carotid ligation. Activated platelets adhere to leukocytes and form circulating mixed aggregates. The latter are considered a reliable marker of a pro-thrombotic state and are associated with several cardiovascular conditions including neointima formation $(39,40)$. CD40/CD40L interactions play an important role in mediating platelet activation and inflammatory processes. Platelets constitutively express CD40 and CD40 ligation leads to enhanced platelet-leukocyte adhesion (41). CD40 on pla- 
telets limits the inflammatory actions of CD40L (42). King et al. showed that mice with an impairment to form platelet-leukocyte aggregates are protected from vascular inflammation and neointimal hyperplasia after vascular injury (40). Hormones released from platelets may act directly on cell types that are involved in the growth of neointima after acute injury. Serotonin has a mitogenic effect on endothelial cells and smooth muscle cells $(43,44)$. Histamine and serotonin increase vascular permeability and ADP increases the agonist-induced oxidative burst in polymorphonuclear leukocytes (45) and amplifies proliferation of smooth muscle cells (44). In line with these data, we show that supernatants of plateletleukocyte aggregates of CD40-deficient mice attenuated SMC proliferation and induced SMC apoptosis in vitro. Therefore, the reduction in platelet-leukocyte aggregates together with the decreased proliferation of SMC after incubation with CD40-deficient platelet-leukocyte aggregates are possible factors leading to decreased neointima formation in CD40-deficient mice. Further experiments will be needed to prove a causal relationship between reduced platelet-leukocyte aggregates and reduced neointima formation in CD40-deficient mice.

In summary, we show that inhibition of the CD40L-Mac interaction does not influence neointima formation. Deficiency of the CD40L binding partner CD40, reduces neointima formation, suggesting that the classic CD40LCD40 interaction mediates neointima formation but not the CD40L-Mac-1 interaction.

\section{Acknowledgements}

We thank Elisa Liehn, at that time member of the laboratory of Prof. Dr. Weber at the IMCAR at the University of Aachen for providing excellent training of the mouse model. We thank Jennifer Buchholz from the University of Freiburg for editorial assistance. This work was supported by research grants from the Deutsche Forschungsgemeinschaft (DFG ZI743/3-1 and 3-2) and from the Else-Kröner-Fresenius Stiftung (EKFS P30/10 // A43/10) to Prof. Dr. Zirlik and grants from the National Health and Medical Research Council of Australia to Prof. Dr. Peter. Prof. Dr. Peter is a Future Fellow of the Australian Research Council.

\section{Conflicts of interest}

None declared.

\section{References}

1. Libby P. Inflammation in atherosclerosis. Nature 2002; 420: 868-874.

2. Donners MM, Daemen MJ, Cleutjens KB, Heeneman S. Inflammation and restenosis: implications for therapy. Ann Med 2003; 35: 523-531.

3. Hassan GS, Merhi Y, Mourad W. CD40 ligand: a neo-inflammatory molecule in vascular diseases. Immunobiology 2011; 217: 521-532.

4. Gerdes N, Zirlik A. Co-stimulatory molecules in and beyond co-stimulation tipping the balance in atherosclerosis? Thromb Haemost 2011; 106: 804-813.

5. Schonbeck U, Libby P. The CD40/CD154 receptor/ligand dyad. Cell Mol Life Sci 2001; 58: 4-43.

6. Mach F, Schonbeck U, Sukhova GK, et al. Functional CD40 ligand is expressed on human vascular endothelial cells, smooth muscle cells, and macrophages: implications for CD40-CD40 ligand signaling in atherosclerosis. Proc Natl Acad Sci USA 1997; 94: 1931-1936.
7. Chai H, Aghaie K, Zhou W. Soluble CD40 ligand induces human coronary artery smooth muscle cells proliferation and migration. Surgery 2009; 146: 5-11.

8. Lutgens E, Daemen MJ. CD40-CD40L interactions in atherosclerosis. Trends Cardiovasc Med 2002; 12: 27-32.

9. Mukundan L, Milhorn DM, Matta B, et al. CD40-mediated activation of vascular smooth muscle cell chemokine production through a Src-initiated, MAPKdependent pathway. Cell Signal 2004; 16: 375-384.

10. Hristov M, Gumbel D, Lutgens E, et al. Soluble CD40 ligand impairs the function of peripheral blood angiogenic outgrowth cells and increases neointimal formation after arterial injury. Circulation 2010; 121: 315-32401/06.

11. Li G, Sanders JM, Bevard MH, et al. CD40 ligand promotes Mac-1 expression, leukocyte recruitment, and neointima formation after vascular injury. Am J Pathol 2008; 172: 1141-1152.

12. Yan JC, Ding S, Liang Y, et al. Relationship between upregulation of CD40 system and restenosis in patients after percutaneous coronary intervention. Acta pharmacologica Sinica 2007; 28: 339-343.

13. Donners MM, Beckers L, Lievens D, et al. The CD40-TRAF6 axis is the key regulator of the CD40/CD40L system in neointima formation and arterial remodeling. Blood 2008; 111: 4596-4604.

14. Song Z, Jin R, Yu S, et al. Crucial Role of CD40 Signaling in Vascular Wall Cells in Neointimal Formation and Vascular Remodeling After Vascular Interventions. Arterioscler Thromb Vasc Biol 2012; 32: 50-64.

15. Song Z, Jin R, Yu S, et al. CD40 is essential in the upregulation of TRAF proteins and NF-kappaB-dependent proinflammatory gene expression after arterial injury. PLoS One 2011; 6: e23239.

16. Stachon P, Missiou A, Walter C, et al. Tumor necrosis factor receptor associated factor 6 is not required for atherogenesis in mice and does not associate with atherosclerosis in humans. PLoS One 2010; 5: e11589.

17. Lutgens E, Lievens D, Beckers L, et al. Deficient CD40-TRAF6 signaling in leukocytes prevents atherosclerosis by skewing the immune response toward an antiinflammatory profile. J Exp Med 2010; 207: 391-404.

18. Leveille C, Bouillon M, Guo W, et al. CD40 ligand binds to alpha5beta1 integrin and triggers cell signaling. J Biol Chem 2007; 282: 5143-5151.

19. Zirlik A, Maier C, Gerdes N, et al. CD40 ligand mediates inflammation independently of CD40 by interaction with Mac-1. Circulation 2007; 115: 1571-1580.

20. Wolf D, Hohmann JD, Wiedemann A, et al. Binding of CD40L to Mac-1's I-domain involves the EQLKKSKTL motif and mediates leukocyte recruitment and atherosclerosis--but does not affect immunity and thrombosis in mice. Circ Res 2011; 109: 1269-1279.

21. Hassan GS, Merhi Y, Mourad W. CD40 ligand: a neo-inflammatory molecule in vascular diseases. Immunobiology 2012; 217: 521-532.

22. Wang Y, Sakuma M, Chen Z, et al. Leukocyte engagement of platelet glycoprotein Ibalpha via the integrin Mac-1 is critical for the biological response to vascular injury. Circulation 2005; 112: 2993-3000.

23. Inoue T, Kato T, Hikichi Y, et al. Stent-induced neutrophil activation is associated with an oxidative burst in the inflammatory process, leading to neointimal thickening. Thromb Haemost 2006; 95: 43-48.

24. Inoue T, Sakai Y, Morooka S, et al. Expression of polymorphonuclear leukocyte adhesion molecules and its clinical significance in patients treated with percutaneous transluminal coronary angioplasty. J Am Coll Cardiol 1996; 28: 1127-1133.

25. Inoue T, Uchida T, Yaguchi I, et al. Stent-induced expression and activation of the leukocyte integrin Mac-1 is associated with neointimal thickening and restenosis. Circulation 2003; 107: 1757-1763.

26. Neumann FJ, Ott I, Gawaz M, et al. Neutrophil and platelet activation at balloon-injured coronary artery plaque in patients undergoing angioplasty. J Am Coll Cardiol 1996; 27: 819-824.

27. Simon DI, Dhen Z, Seifert P, et al. Decreased neointimal formation in Mac-1(-/-) mice reveals a role for inflammation in vascular repair after angioplasty. J Clin Invest 2000; 105: 293-300.

28. Kumar A, Lindner V. Remodeling with neointima formation in the mouse carotid artery after cessation of blood flow. Arterioscler Thromb Vasc Biol 1997; 17: 2238-2244

29. Hilgendorf I, Eisele S, Remer I, et al. The oral spleen tyrosine kinase inhibitor fostamatinib attenuates inflammation and atherogenesis in low-density lipoprotein receptor-deficient mice. Arterioscler Thromb Vasc Biol 2011; 31: 1991-1999. 
30. Missiou A, Rudolf P, Stachon P, et al. TRAF5 deficiency accelerates atherogenesis in mice by increasing inflammatory cell recruitment and foam cell formation. Circ Res 2010; 107: 757-766.

31. Lonsdorf AS, Kramer BF, Fahrleitner M, et al. Engagement of alphaIIbbeta3 (GPIIb/IIIa) with alphanubeta3 integrin mediates interaction of melanoma cells with platelets: a connection to hematogenous metastasis. J Biol Chem 2012; 287: 2168-2178.

32. Willecke F, Zeschky K, Ortiz Rodriguez A, et al. Cannabinoid receptor 2 signaling does not modulate atherogenesis in mice. PLoS One. 2011; 6: e19405.

33. Wolf D, Jehle F, Ortiz Rodriguez A, et al. CD40L deficiency attenuates diet-induced adipose tissue inflammation by impairing immune cell accumulation and production of pathogenic IgG-antibodies. PLoS One 2012; 7: e33026.
34. Andre P, Prasad KS, Denis CV, et al. CD40L stabilizes arterial thrombi by a beta 3 integrin--dependent mechanism. Nat Med 2002; 8: 247-252.

35. Kawai T, Andrews D, Colvin RB, et al. Thromboembolic complications after treatment with monoclonal antibody against CD40 ligand. Nat Med 2000; 6: 114.

36. Hermann A, Schror K, Weber AA. CD40 ligand (CD40L) does not stimulate proliferation of vascular smooth muscle cells. Eur J Cell Biol 2002; 81: 213-221.

37. Remskar M, Li H, Chyu KY, et al. Absence of CD40 signaling is associated with an increase in intimal thickening after arterial injury. Circ Res 2001; 88: 390-394.

38. Eslin DE, Zhang C, Samuels KJ, et al. Transgenic mice studies demonstrate a role for platelet factor 4 in thrombosis: dissociation between anticoagulant and antithrombotic effect of heparin. Blood 2004; 104: 3173-3180.

39. Cerletti C, Tamburrelli C, Izzi B, et al. Platelet-leukocyte interactions in thrombosis. Thromb Res 2012; 129: 263-266.

40. King SM, McNamee RA, Houng AK, et al. Platelet dense-granule secretion plays a critical role in thrombosis and subsequent vascular remodeling in atherosclerotic mice. Circulation 2009; 120: 785-791.

41. Inwald DP, McDowall A, Peters MJ, et al. CD40 is constitutively expressed on platelets and provides a novel mechanism for platelet activation. Circ Res 2003; 92: 1041-1048.

42. Henn V, Steinbach S, Buchner K, et al. The inflammatory action of CD40 ligand (CD154) expressed on activated human platelets is temporally limited by coexpressed CD40. Blood 2001; 98: 1047-1054.

43. Pakala R, Willerson JT, Benedict CR. Mitogenic effect of serotonin on vascular endothelial cells. Circulation 1994; 90: 1919-1926.

44. Hodivala-Dilke KM, McHugh KP, Tsakiris DA, et al. Beta3-integrin-deficient mice are a model for Glanzmann thrombasthenia showing placental defects and reduced survival. J Clin Invest 1999; 103: 229-238.

45. Ruggeri ZM. Platelets in atherothrombosis. Nat Med 2002; 8: 1227-1234. 